Proceedings

\title{
Does the Inhibition of Valosin Containing Protein (VCP) Has an Effect on Apoptosis of Bone Marrow Mesenchymal Stem Cells Derived from Acute Lymphoblastic Leukemia Patients and Healthy Donors? ${ }^{+}$
}

\author{
Şeyma Kipel ${ }^{1}$, Hilal Nakkaş ${ }^{1}$, Sevil Çaylı ${ }^{1}$, Yasin Köksal ${ }^{2}$ and Habibe Meltem Özgüner 1,2,* \\ 1 Department of Histology and Embryology, Ankara Yıldırım Beyazıt University, 06800 Ankara, Turkey; \\ seymakipel@gmail.com (S.K.); gokturkhilal@gmail.com (H.N.); sevilcayli@yahoo.com (S.Ç.) \\ 2 Ankara Child Health and Disease Hematology Oncology Training and Research Hospital, 06080 Ankara, \\ Turkey; yasinkoksal_44@hotmail.com \\ * Correspondence: mozguner@hotmail.com \\ + Presented at the 2nd International Cell Death Research Congress, İzmir, Turkey, 1-4 of November 2018.
}

Published: 6 December 2018

\begin{abstract}
Acute lymphoblastic leukemia (ALL) is the most common cancer type observed in childhood. Bone marrow (BM) microenvironment has significant role both at the beginning and the progress of leukemic period. Mesenchymal stem cells (MSCs) that make up microenvironment, regulate growth factors, cytokines and induction of survival of ALL cells by generating intracellular signals and establishing drug resistance. p97/VCP is a type of protein and it is responsible for also intracellular ubiquitin proteasome pathway, endoplasmic-reticulum-associated protein degradation, cell cycle, apoptosis and autophagy. It is shown that p97/VCP is overexpressed in the most of the cancer types including the ALL. p97/VCP inhibitors serve as therapeutic agents. Amongst p97/VCP inhibitors, DBeQ (dibenzylquinazoline-2,4-diamine) provide to undergo apoptosis and prevents cell proliferation. In this study, MSCs obtained from ALL patients and healthy donors were isolated by density-gradient method and then these cells were cultured. Cells were incubated with DBeQ. Cell apoptosis was determined by Annexin V/PI method (in flow cytometry). According to obtained data, availability of p97/VCP inhibitor which was recommended to be used as therapeutic agent in the treatment of other cancer types, in ALL treatment will be evaluated and this study will provide a basis for future studies.
\end{abstract}

Keywords: Acute lymphoblastic leukemia (ALL); Mesenchymal stem cells (MSCs); apoptosis; DBeQ; p97/VCP

\section{Introduction}

Acute lymphoblastic leukemia (ALL) is a malignant disease that develop from uncontrolled proliferation of $\mathrm{B}$ and $\mathrm{T}$ lymphocyte cells because of mutations occurring in the bone marrow [1]. Mesenchymal stem cells (MSCs) have an important role in the binding of hematopoietic stem cells (HSCs) and secretion of many cytokines [2]. The bone marrow microenvironment provides the selfrenewal and differentiation characters of MSCs. The most important events in the stem cell niche is to maintain the number of stem cells in the niche [3].

p97/Valosin containing protein (VCP) is a member of the AAA + ATPase family. p97/VCP has been working on many important pathway such as protein degradation, cell cyle [4]. But the most 
important features of $\mathrm{p} 97 / \mathrm{VCP}$ are the role of autophagosome maturation, autophagy and apoptosis mechanism [5].

There are many $\mathrm{p} 97 / \mathrm{VCP}$ inhibitors but it has been shown that DBeQ has no toxic effects on the cell and induce cell death mechanism [6].

Apoptosis or programmed cell death is a cell mechanism that is used to destroy unwanted, damaged or potentially neoplastic cells [7]. When the cells enter the apoptosis, the phosphatidylserine present in the membrane of the cells in translocated to the outside of the cell membrane.

The aim of this study was to investigate the effects of DBeQ on viability and apoptosis in MSCs that are obtained from childhood ALL patients and compare the results with healthy donors.

\section{Materials and Methods}

\subsection{Selection of Groups}

BM samples were derived from the patients $(n=4)$ who were diagnosed with ALL. Control groups were selected from healthy volunteer donors $(n=6)$ of patients who applied for allogenic transplantation to BM transplantation unit. After taken approval from healthy donors, sample was taken. The study was approved by the local ethical committee.

\subsection{Collection of Samples}

2-4 mL BM samples of ALL patients aspirated at the time of diagnosis before treatment, were accepted to Stem Cell Processing and Storage Laboratory. In addition, 2-4 mL BM samples of healthy donors were harvested from posterior iliac crest under general anesthesia.

\subsection{Separation of Mononuclear Cells (MNCs) by Density-Gradient Method}

BM samples were mixed with PBS (Phosphate Buffered Saline) (Biochrom, Germany) at a ratio of 1:1 and then they laid on $4 \mathrm{~mL}$ of Biocoll $(1.077 \mathrm{~g} / \mathrm{mL})$ separating solution (Biochrom, Germany). MNCs were separated by centrifugation.

\subsection{Transition from MNCs to MSCS}

MNCs were cultured in $25 \mathrm{~cm}^{2}$ flasks (T25) with Dulbeco's Modified Eagle Medium Low Glucose (DMEM-LG) (Biochrom, Germany), 10\% FBS (Biochrom, Germany) and 1\% penicillinstreptomycin (Biochrom, Germany). The culture media were changed every 3 days. In about two weeks, cells reached confluency of $90 \%$ in culture flasks.

\subsection{Characterization of MSCS}

(a) Morphological analysis: Cells adhere to the plastic surface and show spindle-polygonal morphology by using inverted microscope. (b) Differentiation assays: The differentiation assays of mesenchymal stem cells were performed as indicated in our previous studies [8].

\subsection{Culture of $M S C$ s with $D B e Q$}

$10 \mu \mathrm{M}$ DBeQ, which is inhibitor of p97/VCP, was added to MSCs and incubated for $1 \mathrm{~h}$.

\subsection{Apoptosis Determination by Annexin V/PI}

Cells were acquired on a Beckman Coulter Navios using Kalusa version 1.2 software by using Annexin V FITC/PI apoptosis kit for flow-cytometry.

\section{Results}

Figures 1 and 2 illustrates the data of flow-cytometric analysis. DBeQ was given to the mesenchymal stem cells 2 of 4 patients (Figure 1) and 3 of 6 healthy donors (Figure 2) and they are indicated as $\mathrm{DBeQ}+$ in the figures. Non-given groups are indicated as $\mathrm{DBeQ}-$. 
Patients MSCs ( $\mathrm{n}=4)$

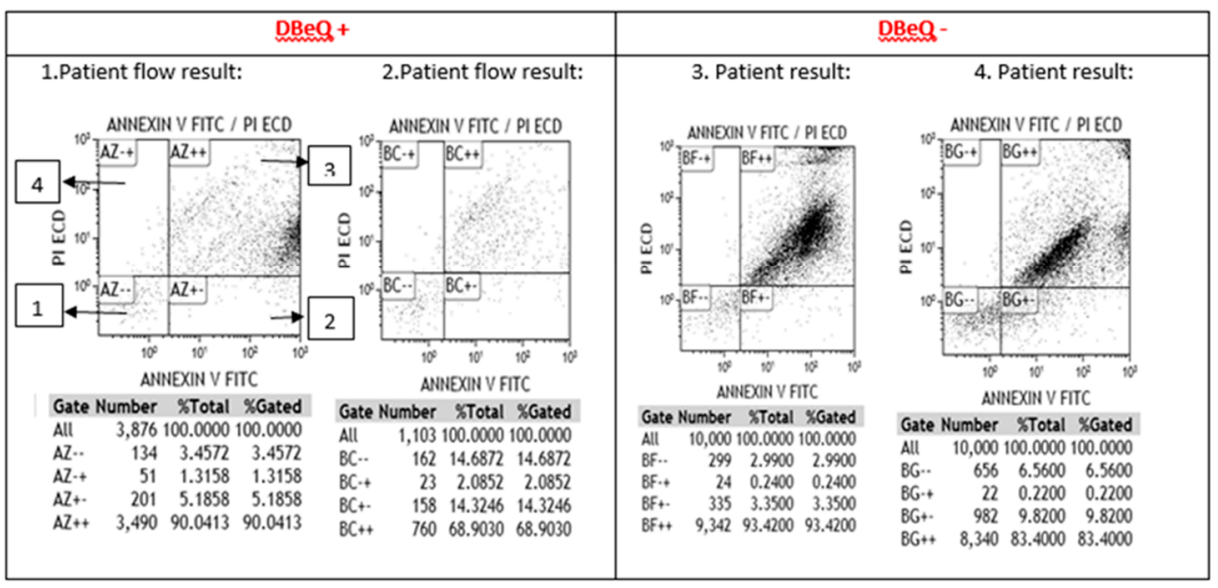

Figure 1. The schematic representation of the graph for apoptosis analysis in flow cytometry is shown in the 1st patient flow results. Field 1 shows live cells. Field 2 shows early apoptotic cells. Field 3 shows late apoptotic. cells. Field 4 shows necrotic cells. Therefore, it is important the results to compare the apoptotic cells after incubating with the inhibitor, to compare the live cells before incubating with the inhibitor.

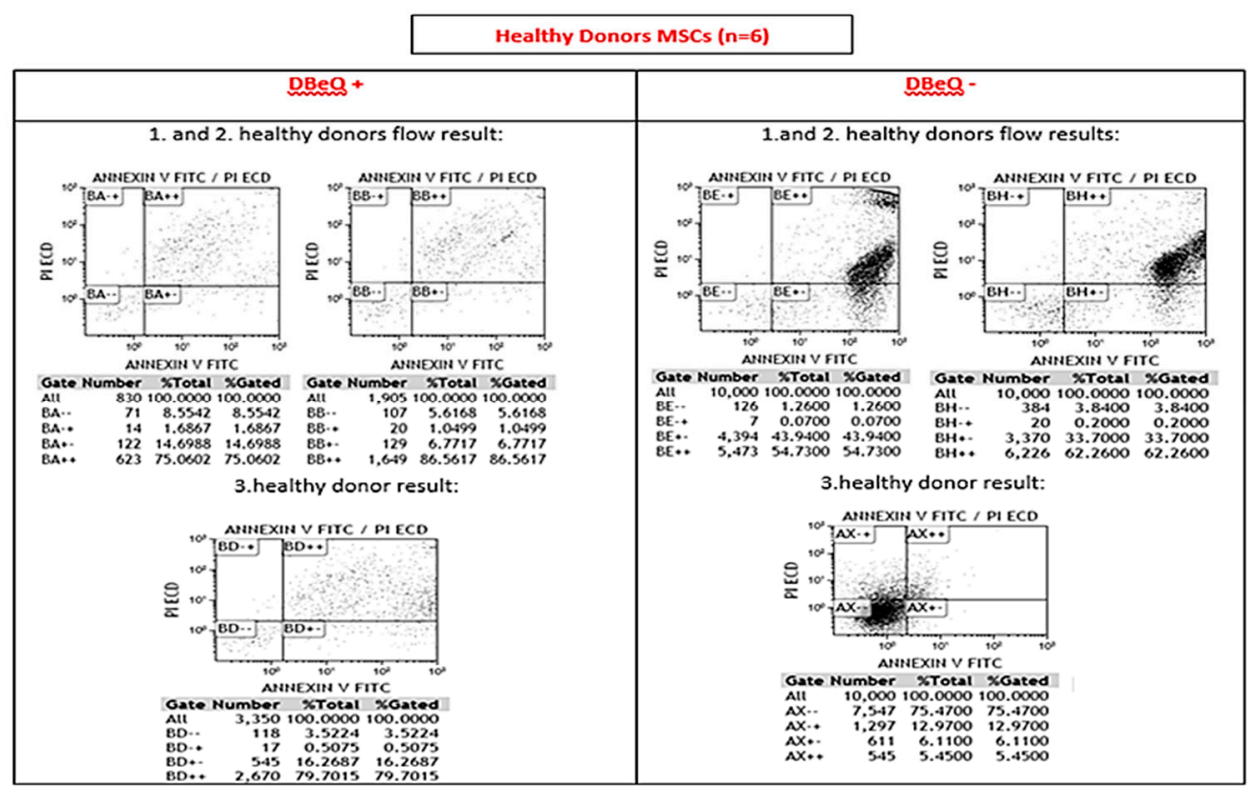

Figure 2. This figure is showed flow analysis results of healthy donor group.

\section{Discussion}

This is the first report concerning the function of $\mathrm{p} 97 / \mathrm{VCP}$ on bone marrow MSCs. In the study we had two groups. The first group BM-MSCs samples were derived from ALL patients $(n=4)$ and the second group BM-MSCs samples were derived from healthy donors $(n=6)$. And then, the groups were subdivided into two which were classified as $\mathrm{DBeQ}-$ and $\mathrm{DBeQ}+$. After an hour incubation period we analyzed the apoptotic cell percantage of the groups.

The percentage of late apoptotic cells in patients BM-MSCs were $93 \%$ and $83 \%$ where as in healthy donors BM-MSCs, these were $54 \%, 62 \%$ and $5 \%$ respectively. We did not analyze this results statistically because two of the results of healthy donors (54\% and $62 \%)$ were relatively higher as expected in normal healthy bone marrow microenvironment. We explain this result as it is possible that we used freezed- thawed samples for two healthy donors BM-MSCs that may change apoptotic 
profiles during these procedures and only one sample that we used freshly, expressed normal apoptotic profile with only $5 \%$ late apoptotic cells. So, we compared the results with only that freshly used sample. And according to results, the patients BM-MSCs in non-inhibitor (DBeQ -) group have increased number of apoptotic cells when compared with healthy donors BM-MSCs. The higher percentage of apoptosis in patients BM-MSCs could be the result of effected bone marrow microenvironment as it is known that malign processes could lead to changes in cell signaling and could create specialized cancer microenvironment with supportive or destructive MSCs [8].

When the results of $\mathrm{DBeQ}$ + groups were compared, the percentage of late apoptotic cells where $90 \%$ and $68 \%$ in ALL patients BM-MSCs and 75\%, 86\%, 79\% in healthy donors BM-MSCs. The percentage of apoptotic cells in all $\mathrm{DBeQ}+$ groups were significantly higher than $\mathrm{DBeQ}$ - healthy donors.

In summary, the present study, investigating the apoptotic profiles of BM-MSCs in ALL patients and healthy donors revealed that DBeQ inhibition of p97/VCP in culture conditions induce apoptosis especially in healthy donors BM-MSCs therefore; as ALL patients BM-MSCs have increased apoptotic activity $\mathrm{DBeQ}$ only support the apoptotic condition. These preliminary results suggest a possible role of p97/VCP on apoptotic pathway of BM-MSCs. Further studies may provide molecular insight into the roles of $\mathrm{p} 97 / \mathrm{VCP}$ in BM-MSCs even in healthy and pathologic conditions.

Author Contributions: H.M.Ö. designed the study; H.M.Ö. and Ş.K. wrote the manuscript; H.M.Ö, Ş.K., S.Ç. and Y.K. provided essential materials/reagents; S..K. and Y.K. performed the experiments. H.M.Ö., Ş.K., H.N., S.Ç. and Y.K. analyzed and discussed the data. All authors read approved the final manuscript.

Acknowledgments: Financial disclosure statement: There is no nothing to disclose.

Conflicts of Interest: The authors declare that they have no conflict of interest.

\section{References}

1. Uzunhan, T.A.; Karakaş, Z. Çocukluk Çağı Akut Lenfoblastik Lösemisi. Çocuk Derg. 2012, 12, 6-15.

2. Lakshmipathy, U.; Verfaillie, C. Stem cell plasticity. Blood Rev. 2005, 19, 29-38.

3. Can, A. Kök Hücre Biyolojisi, Türleri ve Tedavisinde Kullanımları, 1 ed.; Akademisyen Tıp Kitapevi: Ankara, Turkey, 2014; pp. 39-46, 175-194, 363-337.

4. Neuwald, A.F.; Aravind, L.; Spouge, J.L.; Koonin, E. AAA+: A class of chaperone-like ATPases associated with the assembly, operation, and disassembly of protein complexes. Genome Res. 1999, 1, $27-43$.

5. Yeo, B.; Hong, C.; Chung, K.; Woo, H.; Kim, K.; Jung, S.; Kim, E.; Yu, S. Valosin-containing protein is a key mediator between autophagic cell death and apoptosis in adult hippocampal neural stem cells following insulin withdrawal. Mol. Brain 2016, 9, 31.

6. Chou, T.; Deshaies, R. Development of p97 AAA ATPase inhibitors. Landes Biosci. 2011, 10, 91-92.

7. Wickremasinghe, R.; Hoffbrand, V. Biochemical and genetic control of apoptosis: Relevance to normal hematopoiesis and hematological malignancies. Blood 1999, 11, 3587-3600.

8. Ok Bozkaya, I.; Azik, F.; Tavil, B.; Koksal, Y.; Ozguner, M.; Tunc, B.; Uckan Cetinkaya, D. The Effect of Granulocyte Colony-Stimulating Factor on Immune-Modulatory Cytokines in the Bone Marrow Microenvironment and Mesenchymal Stem Cells of Healthy Donors. Biol. Blood Marrow Transplant. 2015, $21,1888-1894$.

(C) 2018 by the authors. Licensee MDPI, Basel, Switzerland. This article is an open access article distributed under the terms and conditions of the Creative Commons Attribution (CC BY) license (http://creativecommons.org/licenses/by/4.0/). 